\title{
Thickness of carotid intima and epicardial fat in rosacea: a cross-sectional study ${ }^{\star}$
}

\author{
Asli Akin Belli ${ }^{1}$ \\ Ibrahim Altun ${ }^{3}$ \\ DOI: http://dx.doi.org/10.1590/abd1806-4841.20176832
}

Ilknur Altun ${ }^{2}$

\begin{abstract}
BACKGROUND: Rosacea is a chronic facial skin disease associated with excessive inflammatory response to various triggers. Although some studies have supported the increased risk of cardiovascular diseases in rosacea, it has not been completely accepted.

OвJестіvE: We aimed to investigate epicardial fat thickness and carotid intima-media thickness as cardiovascular risk predictors in rosacea patients.

METHODS: We conducted a cross-sectional study including 40 rosacea patients and 40 controls. Demographic data, epicardial fat thickness, carotid intima-media thickness, lipid parameters, biochemical parameters, presence of insulin resistance, and presence of metabolic syndrome of the participants were recorded.

RESULTS: Forty rosacea patients ( 31 female and 9 male) and 40 controls ( 30 female and 10 male) were enrolled in the study. Rosacea patients had significantly higher epicardial fat thickness and carotid intima-media thickness volumes than controls $(\mathrm{P}<0.001)$. In the multivariate logistic regression analysis, epicardial fat thickness was independently related to presence of rosacea (P<0.001, $\mathrm{OR}=13.31)$. In the multiple linear regression analysis, the epicardial fat thickness was independently associated with rosacea $(\beta=0.47, P<0.001)$, carotid intima-media thickness $(\beta=0.36, P<0.001)$, and systolic blood pressure $(\beta=0.19, P=0.015)$ and the carotid intima-media thickness was independently associated with epicardial fat thickness $(\beta=0.6, P<0.001)$. The epicardial fat thickness levels were correlated with carotid intima-media thickness ( $r=0.63, P<0.001)$, LDL ( $r=0.23, P=0.037)$, systolic blood pressure $(\mathrm{r}=0.45, \mathrm{P}<0.001)$, and diastolic blood pressure levels $(\mathrm{r}=0.37, \mathrm{P}=0.001)$. The carotid intima-media thickness levels were correlated with epicardial fat thickness $(\mathrm{r}=0.63, \mathrm{P}<0.001)$, systolic blood pressure $(\mathrm{r}=0.04, \mathrm{P}<0.001)$, and diastolic blood pressure levels $(\mathrm{r}=0.27, \mathrm{P}=0.016)$.
\end{abstract}

STUDY LIMITATIONS: The small number of participants.

CONCLUSIONS: Examination and follow-up of rosacea patients for cardiovascular diseases may be recommended practices. Keywords: Cardiovascular diseases; Risk factors; Rosacea

\section{INTRODUCTION}

Rosacea is a chronic facial skin disease associated with aberrant inflammatory response and vascular factors. Even though the relation of rosacea to cardiovascular diseases, as well as mortality due to cardiovascular events have been emphasized in recent studies, this relationship has not been completely accepted. Whereas Egeberg et al. have reported no increased risk of mortality due to cardiovascular diseases in rosacea patients, Duman et al. have reported high cardiovascular risk factors in rosacea patients. ${ }^{1,2}$ Furthermore, we have found the rate of insulin resistance increased in rosacea patients in our previous study. ${ }^{3}$
Recently, measurement of epicardial fat thickness (EFT) and carotid intima-media thickness (CIMT) have become popular to indicate the risk of cardiovascular disease and subclinical atherosclerosis as non-invasive and practical methods. It has been shown that EFT and CIMT have been increased in patients with metabolic syndrome, insulin resistance, and major cardiac events in several studies. ${ }^{4-6}$

Since there is a well-known association between psoriasis and cardiometabolic risk factors, EFT and CIMT have been investigated in patients with psoriasis and these parameters have been found to be increased in some studies. ${ }^{7-9}$ Although a precise relationship between

\footnotetext{
Received on 15.12.2016.

Approved by the Advisory Board and accepted for publication on 22.04.2017.

*Work performed at the Mugla Sitki Kocman University Training and Research Hospital, Department of Dermatology - Muğla, Turkey

Financial support: None.

Conflict of interest: None

Department of Dermatology, Mugla Sitki Kocman University Training and Research Hospital - Muğla, Turkey

Department of Radiology, Mugla Sitki Kocman University Training and Research Hospital - Muğla, Turkey.

Department of Cardiology, Mugla Sitki Kocman University Medical School - Muğla, Turkey.

(C2017 by Anais Brasileiros de Dermatologia
} 
rosacea and cardiovascular diseases as in psoriasis has not been shown, rosacea has also chronic course and inflammatory pathogenesis. To the best of our knowledge, there is no study investigating EFT and CIMT in rosacea patients to predict cardiovascular events early and to assess the necessity of cardiovascular examination.

We aimed to investigate EFT and CIMT in rosacea patients compared with controls in this pilot study.

\section{METHODS}

We conducted a cross-sectional study including 40 rosacea patients and 40 age-, gender-, and body mass index (BMI)- matched controls in the Dermatology Department of Mugla Sitki Kocman University Training and Research Hospital between January 2016 and October 2016. We obtained the Ethic Committee Approval before the study. Rosacea and control groups were selected consecutively from the patients who meet the inclusion/exclusion criteria. The diagnosis of rosacea was based on the National Rosacea Society criteria. Our exclusion criteria for rosacea patients and controls were: presence of a known cardiovascular disease, diabetes mellitus, cerebrovascular disease, peripheral vascular disease, any other inflammatory disease, and pregnancy.

We recorded demographic data (age and gender), data related to rosacea (duration, subtype, and localization), smoking history, alcohol consumption, history of regular exercise, family history of cardiovascular disease (CVD), anthropometric measures, lipid parameters, fasting blood glucose (FBG), basal insulin levels, systolic blood pressure, and diastolic blood pressure levels of the participants. BMI was calculated using the formula weight $(\mathrm{kg})$ / height ${ }^{2}\left(\mathrm{~m}^{2}\right)$. All biochemical blood parameters were studied after a 12-hour fasting period. The homeostasis model assessment of insulin resistance (HOMA-IR) was used to calculate insulin resistance according to the following formula:

"Fasting insulin level $(\mu \mathrm{UI} / \mathrm{mL}) \times$ Fasting blood glucose level $(\mathrm{mg} / \mathrm{dL}) / 405$ ". A value of $>2.7$ was considered indicative of IR.

Based on the diagnostic criteria of the International Diabetes Federation (IDF-2005), waist circumference $>94 \mathrm{~cm}$ in men and $>80 \mathrm{~cm}$ in women, plus at least two of the following criteria: triglyceride value $>150 \mathrm{mg} / \mathrm{dL}$ or specific treatment for this lipid abnormality, high density lipoprotein $<40 \mathrm{mg} / \mathrm{dL}$ in men and $<50$ $\mathrm{mg} / \mathrm{dL}$ in women or specific treatment for this lipid abnormality, blood pressure $\geq 130 / 85 \mathrm{mmHg}$ or antihypertensive treatment, fasting blood glucose $\geq 100 \mathrm{mg} / \mathrm{dL}$ or diagnosed diabetes mellitus, were accepted as metabolic syndrome. ${ }^{10}$

A single cardiologist performed standard transthoracic echocardiography (TTE) in all patients using a Philips EPIQ 7 ultrasonography system (Koninklijke Philips N.V.; Best, The Netherlands). Epicardial fat was determined between the visceral layer of the pericardium and the outer wall of the myocardium as the relatively echo-free space. EFT was measured in end-diastole on the free wall of the right ventricle in the parasternal long- and short-axis views. ${ }^{11}$ The average value of the maximal values measured at any site was considered.

A single radiologist performed carotid ultrasound in all patients by using a high-resolution B mode Doppler ultrasonography
(Aplio 500, Toshiba, Japan) with a $7.5 \mathrm{mHz}$ linear-array transducer. CIMT was defined as the distance between lumen-intima and the media-adventitia of the carotid arterial wall on the ultrasound. Measurements were performed at the level of the common carotid artery bulbus - $1 \mathrm{~cm}$ proximal from the bifurcation. The average value of two measurements of the right and left carotid arteries was considered. ${ }^{12}$ Both of the cardiologist and radiologist were unaware of the participants' status (rosacea patient or control).

For the data analysis, the statistical program "SPSS for windows 22.0" was employed. For descriptive statistics of the data, we have used mean, standard deviation, ratio and frequency. The distribution of variables was checked with Kolmogorov-Smirnov test; independent samples $\mathrm{T}$ test and Mann-Whitney U-test were used for variables distributed normally and non-normally, respectively. The chi-square test was used for the analysis of qualitative data. Multivariate logistic regression analysis was performed with rosacea as dependent variable. In addition, multivariate linear regression analysis was performed separately with EFT and CIMT as dependent variables. Variables which showed $\mathrm{P}<0.1$ in univariate analysis were included in the multivariate logistic and linear regression model. Since EFT and CIMT were collinear, one of them was selected for the multiple regression analysis. Similarly, since systolic blood pressure and diastolic blood pressure were collinear, one of them was selected for the multivariate linear regression analysis. Correlation of the variables with epicardial fat thickness (EFT) and carotid intima media thickness (CIMT) was checked with Pearson correlation analysis. $\mathrm{P}<0.05$ was assessed as significant.

\section{RESULTS}

Forty rosacea patients ( 31 females, 9 males; age range 37-68 years, mean 50.35) and 40 controls ( 30 females, 10 males; age range $35-70$ years, mean 50.52) were included in the study. The mean rosacea duration was 3.93 years (range 3 months to 20 years). Twenty-two patients (55\%) had erythematotelangiectatic type and 18 patients $(45 \%)$ had papulopustular type rosacea.

Rosacea patients had significantly higher EFT (Cohen's $d$ 1.7; effect size $r$ - 0.66; 95\% CI- 0.84-1.39) and CIMT volumes (Cohen's d- 0.8; effect size r- 0.39; 95\% CI- 0.05-0.15) than controls (P $<0.001$ ). Additionally, the levels of systolic blood pressure and diastolic blood pressure were significantly higher than in the control group $(\mathrm{P}<0.05)$ (Table 1$)$.

In the multivariate logistic regression analysis, only EFT was independently related to the presence of rosacea $(\mathrm{P}<0.05)$ (Table 2).

In the multiple linear regression analysis in which EFT was included as a dependent variable with rosacea, CIMT, LDL, and systolic blood pressure, the EFT was independently associated with rosacea, CIMT, and systolic blood pressure level $(\mathrm{P}<0.05)$ (Table 3). In the multiple linear regression analysis in which CIMT was included as a dependent variable with rosacea, EFT, and systolic blood pressure, the CIMT was independently associated with EFT $(\mathrm{P}<0.001)$ (Table 4).

In addition, the EFT levels were correlated with the CIMT, LDL, systolic blood pressure, and diastolic blood pressure levels ( $P$ $<0.05$ ). The CIMT levels were correlated with the EFT, systolic blood pressure, and diastolic blood pressure levels $(\mathrm{P}<0.05)$ (Table 5). 


\begin{tabular}{|c|c|c|c|}
\hline & $\begin{array}{l}\text { Rosacea group }(n=40) \\
n(\%) / \text { mean } \pm S D\end{array}$ & $\begin{array}{l}\text { Control group }(n=40) \\
n(\%) / \text { mean } \pm S D\end{array}$ & $\mathbf{P}$ \\
\hline Age (years) & $50.35 \pm 7.59$ & $50.52 \pm 7.96$ & 0.867 \\
\hline Smoking & $4(10 \%)$ & $6(15 \%)$ & 0.499 \\
\hline Alcohol consumption & $2(5 \%)$ & $2(5 \%)$ & 1 \\
\hline Regular exercise & $16(40 \%)$ & $23(57.5 \%)$ & 0.117 \\
\hline Family history of CVD & $5(12.5 \%)$ & $6(15 \%)$ & 0.815 \\
\hline EFT (mm) & $4.46 \pm 0.65$ & $3.28 \pm 0.59$ & $<0.001$ \\
\hline CIMT (mm) & $0.72 \pm 0.19$ & $0.61 \pm 0.12$ & $<0.001$ \\
\hline LDL (mg/dL) & $133.74 \pm 33.39$ & $121.76 \pm 24.68$ & 0.058 \\
\hline TG (mg/dL) & $141.45 \pm 75.06$ & $123.24 \pm 59.11$ & 0.177 \\
\hline Total Cholesterol (mg/dL) & $215.54 \pm 40.78$ & $206.31 \pm 30.45$ & 0.216 \\
\hline HDL (mg/dL) & $53.89 \pm 12.31$ & $59.87 \pm 16.98$ & 0.067 \\
\hline FBG $(\mathrm{mg} / \mathrm{dL})$ & $95.97 \pm 14.96$ & $94.49 \pm 11.46$ & 0.791 \\
\hline CRP (mg/dL) & $3.16 \pm 3.16$ & $2.26 \pm 1.99$ & 0.090 \\
\hline BMI $\left(\mathrm{kg} / \mathrm{m}^{2}\right)$ & $27.75 \pm 3.08$ & $27.14 \pm 3.52$ & 0.597 \\
\hline Systolic BP (mm Hg) & $123.14 \pm 16.94$ & $114.36 \pm 14.11$ & 0.045 \\
\hline Diastolic BP (mm Hg) & $80 \pm 10.57$ & $74.61 \pm 6.82$ & 0.016 \\
\hline HOMA-IR & $2.41 \pm 1.31$ & $2.14 \pm 1.19$ & 0.329 \\
\hline HOMA-IR > 2.7 & $14(35 \%)$ & $10(25 \%)$ & 0.329 \\
\hline MS & $13(32.5 \%)$ & $8(20 \%)$ & 0.204 \\
\hline
\end{tabular}

Chi-square test, Independent samples t test, and Mann-Whitney U-test. SD- Standard deviation; CVD- Cardiovascular disease; EFT- Epicardial fat thickness; CIMT- Carotid intima-media thickness; TG-Triglyceride; FBG- Fasting blood glucose; BMI- Body mass index; BP- Blood pressure; HOMA-IR- Homeostasis model assessment of insulin resistance; MS- Metabolic syndrome.

\begin{tabular}{|c|c|c|c|c|}
\hline Variables & Univariate analysis $P$ & Odds Ratio (95\% CI) & Multivariate analysis $\beta \mathbf{P}$ & Odds Ratio (95\% CI) \\
\hline Gender & 0.793 & $1.15(0.41-3.22)$ & & \\
\hline Age & 0.865 & $1.01(0.95-1.06)$ & & \\
\hline Smoking & 0.502 & $1.59(0.41-6.12)$ & & \\
\hline Alcohol consumption & 1 & $1(0.13-7.47)$ & & \\
\hline Regular exercise & 0.119 & $2.03(0.83-4.95)$ & & \\
\hline Family history of CVD & 0.815 & $0.86(0.24-3.09)$ & & \\
\hline Waist circumference & 0.911 & $1.01(0.957-1.05)$ & & \\
\hline EFT & $<0.001$ & $12.87(4.43-37.36)$ & $2.59<0.001$ & $13.31(4.15-42.68)$ \\
\hline LDL & 0.062 & $1.02(0.99-1.03)$ & 0.010 .534 & $1.01(0.98-1.03)$ \\
\hline TG & 0.180 & $1.01(0.99-1.01)$ & & \\
\hline Total cholesterol & 0.215 & $1.01(0.99-1.02)$ & & \\
\hline HDL & 0.073 & $0.97(0.94-1.01)$ & -0.050 .087 & $0.96(0.91-1.01)$ \\
\hline FBG & 0.413 & $1.01(0.98-1.05)$ & & \\
\hline CRP & 0.124 & $1.18(0.95-1.46)$ & & \\
\hline Diastolic BP & 0.014 & $1.08(1.02-1.15)$ & 0.050 .291 & $1.05(0.96-1.15)$ \\
\hline BMI & 0.592 & $1.04(0.91-1.19)$ & & \\
\hline IR & 0.331 & $1.61(0.61-4.25)$ & & \\
\hline MS & 0.207 & $1.93(0.69-5.34)$ & & \\
\hline
\end{tabular}

CI- Confidence interval; CVD- Cardiovascular disease; EFT- Epicardial fat thickness; TG-Triglyceride; FBG- Fasting blood glucose; CRP- C-reactive protein; BP- Blood pressure; BMI- Body mass index; IR- Insulin resistance; MS- Metabolic syndrome. 


\begin{tabular}{|c|c|c|}
\hline Variables & Univariate analysis $P$ & $\begin{array}{l}\text { Multivariate } \\
\text { analysis } \beta P\end{array}$ \\
\hline Rosacea & $<0.001$ & $0.47<0.001$ \\
\hline Gender & 0.564 & \\
\hline Age & 0.199 & \\
\hline Duration of rosacea & 0.458 & \\
\hline Subtype of rosacea & 0.763 & \\
\hline Smoking & 0.267 & \\
\hline Alcohol consumption & 0.75 & \\
\hline Regular exercise & 0.427 & \\
\hline Family history of CVD & 0.572 & \\
\hline Waist circumference & 0.285 & \\
\hline CIMT & $<0.001$ & $0.36<0.001$ \\
\hline LDL & 0.037 & 0.060 .388 \\
\hline TG & 0.971 & \\
\hline Total cholesterol & 0.118 & \\
\hline HDL & 0.742 & \\
\hline FBG & 0.697 & \\
\hline CRP & 0.824 & \\
\hline Systolic BP & $<0.001$ & 0.190 .015 \\
\hline BMI & 0.814 & \\
\hline IR & 0.448 & \\
\hline MS & 0.908 & \\
\hline
\end{tabular}

CIMT- Carotid intima media thickness; CVD- Cardiovascular disease; TG-Triglyceride; FBGFasting blood glucose; CRP- C-reactive protein; BP- Blood pressure; BMI- Body mass index; IR- Insulin resistance; MS- Metabolic syndrome.

TABLE 5: Correlation of the variables with epicardial fat thickness (EFT) and carotid intima-media thickness (CIMT)

\begin{tabular}{lll} 
Variables & EFT CC P & CIMT CC P \\
\hline Age & 0.1450 .199 & 0.1460 .197 \\
Waist circumference & 0.1230 .285 & 0.0410 .724 \\
EFT & 1 & $0.629<0.001$ \\
CIMT & $0.629<0.001$ & 1 \\
LDL & 0.2340 .037 & 0.1240 .273 \\
TG & -0.0040 .971 & 0.0590 .601 \\
Total cholesterol & 0.1760 .118 & 0.1280 .258 \\
HDL & -0.0370 .742 & 0.0040 .969 \\
FBG & 0.0440 .697 & 0.1120 .321 \\
CRP & 0.0260 .824 & -0.0050 .967 \\
Systolic BP & $0.447<0.001$ & $0.371<0.001$ \\
Diastolic BP & 0.3690 .001 & 0.2720 .016 \\
BMI & 0.0270 .814 & -0.0370 .748 \\
HOMA-IR & 0.0620 .583 & 0.0520 .647 \\
\hline
\end{tabular}

EFT- Epicardial fat thickness; CIMT- Carotid intima media thickness; CC- Correlation coefficient; TG-Triglyceride; FBG- Fasting blood glucose; CRP- C-reactive protein; BPBlood pressure; BMI- Body mass index; HOMA-IR- Homeostasis model assessment of insulin resistance.
TABLE 4: Independent predictors for carotid intima-media thickness by multivariate linear regression analysis

\begin{tabular}{lll} 
Variables & Univariate analysis P & $\begin{array}{l}\text { Multivariate } \\
\text { analysis } \beta \mathbf{P}\end{array}$ \\
\hline Rosacea & $<0.001$ & -0.030 .814 \\
Gender & 0.261 & \\
Age & 0.197 & \\
Duration of rosacea & 0.579 & \\
Subtype of rosacea & 0.984 & \\
Smoking & 0.859 & \\
Alcohol consumption & 0.898 & \\
Regular exercise & 0.936 & \\
Family history of CVD & 0.519 & \\
Waist circumference & 0.724 & \\
EFT & $<0.001$ & \\
LDL & 0.273 & \\
TG & 0.601 & \\
Total cholesterol & 0.258 & \\
HDL & 0.969 & \\
FBG & 0.321 & \\
CRP & 0.967 & \\
Systolic BP & 0.001 & \\
BMI & 0.748 & \\
IR & 0.289 & \\
MS & 0.119 & \\
\hline
\end{tabular}

CVD- Cardiovascular disease; EFT- Epicardial fat thickness; TG-Triglyceride; FBG- Fasting blood glucose; CRP- C-reactive protein; BP- Blood pressure; BMI- Body mass index; IR- Insulin resistance; MS- Metabolic syndrome.

\section{DISCUSSION}

Rosacea is a common cutaneous disease with the frequent involvement of cheek, nose, forehead, and chin. Although it is known to be a chronic inflammatory disease, ambiguity associated with the pathogenesis is still continuing. Whereas the relation of rosacea to cardiovascular diseases has been emphasized in recent years, some new studies have reported no association between these diseases. The diverse results have encouraged us to conduct this pilot study. We found the volumes of EFT and CIMT increased in rosacea patients when compared to controls.

Epicardial fat tissue, a type of visceral adipose tissue, surrounds the coronary vessels and heart, particularly atrioventricular and interventricular grooves. Evaluation of EFT and CIMT have been stated to show cardiac adiposity and subclinical atherosclerosis, respectively. EFT acts as an endocrine organ and can induce coronary artery disease by secreting various proinflammatory cytokines, chemokines, and hormones including tumor necrosis factor- a (TNF-a), plasminogen activator inhibitor-1, IL-6, monocyte chemoattractant protein-1 (MCP-1), and leptin. Thus, measurement of EFT and CIMT has been proposed as a new predictor of cardiometabolic diseases. In various studies, EFT and CIMT have been found increased in the patients with insulin resistance, major cardiac events, complex coronary artery disease, acute ischemic stroke, 
and diabetes mellitus.-6,13-15 Moreover, Hwang et al reported that EFT is related to the formation of non-calcified coronary plaque in asymptomatic individuals. ${ }^{16}$ Similarly, it has been stated that EFT is associated with atherosclerotic plaque and CIMT by Kocaman et al. ${ }^{17}$ In our study, EFT and CIMT were also strongly correlated.

In the pathogenesis of rosacea, increased inflammatory response to various triggers associated with cathelicidin peptides and endoplasmic reticulum stress have been demonstrated. Pro-inflammatory cytokines such as IL- 8 , IL- $1 \beta$, and TNF- $\alpha$ and inflammasome-related genes have been found overexpressed in the facial skin of rosacea patients. ${ }^{18-20}$ Firstly, the relation of rosacea to cardiovascular diseases have been investigated by Duman et al. and cardiovascular risk factors have been found increased in rosacea patients. ${ }^{2}$ In another study, dyslipidemia and hypertension have been reported increased in rosacea patients. ${ }^{21}$ Another study reported that rosacea patients had more dyslipidemia and hypertension than patients without rosacea. Rainer et al. have noted that the risk of cardiovascular diseases, metabolic diseases, and gastroesophageal reflux disease are increased in the patients with moderate-severe rosacea than in the patients with mild rosacea. ${ }^{22}$ In our previous study, the rate of insulin resistance and levels of FBG, total cholesterol, systolic blood pressure, and diastolic blood pressure were significantly higher in rosacea patients than in controls. ${ }^{3}$ However, Egeberg et al. have reported no increased risk of mortality due to cardiovascular events in rosacea patients in their two studies. ${ }^{1,23}$ These conflicting results encouraged us to deepen this topic. In the current study, EFT and CIMT levels were significantly higher in rosacea patients than in controls. Additionally, the other cardiovascular risk factors including systolic blood pressure and diastolic blood pressure levels were significantly higher in the rosacea group than in the control group. The current results may be explained by that decreased activity of paraoxone-1 (PON1), an antioxidant enzyme, increased cathelicidin gene expression; similar pro-inflammatory pathways have been shown in atherosclerosis and cardiovascular diseases. ${ }^{24-27}$ Among the other chronic cutaneous inflammatory diseases, EFT and CIMT have been evaluated in psoriasis patients in a few studies. Torres et al. have compared EFT, abdominal visceral fat, and coronary artery calcification in 100 psoriasis patients and 202 controls and found significantly increased EFT volume and subclinical atherosclerosis in psoriasis patients. ${ }^{7}$ In another study, Bacaksiz et al. have reported that EFT was significantly increased in psoriasis patients, particularly with severe disease. ${ }^{8}$ Bulbul Sen et al. have found EFT and CIMT levels increased in 65 psoriasis patients compared to 50 controls and have noticed that EFT could be a practical marker to indicate subclinical atherosclerosis and high cardiovascular risk in psoriasis patients. ${ }^{9}$ Although there is no precise relationship between rosacea and cardiovascular diseases as in psoriasis, rosacea is also a chronic inflammatory disease.

To exclude the bias due to obesity, we enrolled age-, gender, and also BMI-matched individuals in both groups. However, our limitations were the small number of the participants in each group and use of trans-thoracic echocardiography to measure EFT rather than magnetic resonance imaging (MRI), which is the gold standard for the measurement of EFT.

\section{CONCLUSION}

In conclusion, the volumes of EFT and CIMT were significantly higher in the rosacea group than in the control group and strongly correlated with each other. According to the current results and some previous studies, examination and follow-up of rosacea patients for developing cardiovascular diseases with biochemical tests and cardiology consultation may be recommended clinical practices. However, our results should be confirmed with a larger number of patients in future studies. 


\section{REFERENCES}

1. Egeberg A, Hansen PR, Gislason GH, Thyssen JP. Assessment of the risk of cardiovascular disease in patients with rosacea. J Am Acad Dermatol. 2016;75:336-9.

2. Duman N, Ersoy Evans S, Atakan N. Rosacea and cardiovascular risk factors: a case control study. J Eur Acad Dermatol Venereol. 2014;28:1165-9.

3. Akin Belli A, Ozbas Gok S, Akbaba G, Etgu F, Dogan G. The relationship between rosacea and insulin resistance and metabolic syndrome. Eur $\mathrm{J}$ Dermatol. 2016;26:260-4.

4. Pierdomenico SD, Pierdomenico AM, Cuccurullo F, lacobellis G. Meta-analysis of the relation of echocardiographic epicardial adipose tissue thickness and the metabolic syndrome. Am J Cardiol. 2013;111:73-8.

5. Altin C, Sade LE, Gezmis E, Yilmaz M, Ozen N, Muderrisoglu H. Assessment of epicardial adipose tissue and carotid/femoral intima media thickness in insulin resistance. J Cardiol. 2017;69:843-850.

6. Tanindi A, Erkan AF, Ekici B. Epicardial adipose tissue thickness can be used to predict major adverse cardiac events. Coron Artery Dis. 2015;26:686-91.

7. Torres T, Bettencourt N, Mendonça D, Vasconcelos C, Gama V, Silva BM, et al. Epicardial adipose tissue and coronary artery calcification in psoriasis patients. J Eur Acad Dermatol Venereol. 2015;29:270-7.

8. Bacaksız A, Tasal A, Sevgili E, Erdoğan E, Onsun N, Sönmez O, et al. Epicardial fat thickness in patients with psoriasis vulgaris. Turk Kardiyol Dern Ars. 2014:42:47-54.

9. Bulbul Sen B, Atci N, Rifaioglu EN, Ekiz O, Kartal I, Buyukkaya E, et al. Increased epicardial fat tissue is a marker of subclinical atherosclerosis in patients with psoriasis. Br J Dermatol. 2013;169:1081-6.

10. Alberti KG, Zimmet P, Shaw J; IDF Epidemiology Task Force Consensus Group. The metabolic syndrome-a new worldwide definition. Lancet. 2005;366:1059-62.

11. lacobellis G, Willens HJ. Echocardiographic epicardial fat: a review of research and clinical applications. J Am Soc Echocardiogr. 2009;22:1311-9.

12. Touboul PJ, Hennerici MG, Meairs S, Adams H, Amarenco P, Bornstein N, et al. Mannheim carotid intima-media thickness and plaque consensus (2004-20062011). An update on behalf of the advisory board of the 3rd, 4th and 5th watching the risk symposia, at the 13th, 15th and 20th european stroke conferences, Mannheim, Germany, 2004, Brussels, Belgium, 2006, and Hamburg, Germany, 2011. Cerebrovasc Dis. 2012;34:290-6.

13. Altun I, Unal Y, Basaran O, Akin F, Emir GK, Kutlu G, et al. Increased Epicardial Fat Thickness Correlates with Aortic Stiffness and N-Terminal Pro-Brain Natriuretic Peptide Levels in Acute Ischemic Stroke Patients. Tex Heart Inst J. 2016;43:220-6.

14. Song DK, Hong YS, Lee H, Oh JY, Sung YA, Kim Y. Increased Epicardial Adipose Tissue Thickness in Type 2 Diabetes Mellitus and Obesity. Diabetes Metab J. 2015;39:405-13.
15. Salazar J, Luzardo E, Mejías JC, Rojas J, Ferreira A, Rivas-Ríos JR, et al. Epicardial Fat: Physiological, Pathological, and Therapeutic Implications. Cardiol Res Pract. 2016;2016:1291537.

16. Hwang IC, Park HE, Choi SY. Epicardial Adipose Tissue Contributes to the Development of Non-Calcified Coronary Plaque: A 5-Year Computed Tomography Follow-up Study. J Atheroscler Thromb. 2017;24:262-274.

17. Kocaman SA, Baysan O, Cetin M, Kayhan Altuner T, Polat Ocaklı E, Durakoğlugil $\mathrm{ME}$, et al. An increase in epicardial adipose tissue is strongly associated with carotid-intima media thickness and atherosclerotic plaque, but LDL only with the plaque. Anatol J Cardiol. 2017;17:56-63.

18. Yamasaki K, Gallo RL. Rosacea as a Disease of Cathelicidins and Skin Innate Immunity. J Investig Dermatol Symp Proc. 2011;15:12-5.

19. Melnik BC. Endoplasmic reticulum stress: key promoter of rosacea pathogenesis Exp Dermatol. 2014;23:868-73.

20. Casas C, Paul C, Lahfa M, Livideanu B, Lejeune 0, Alvarez-Georges S, et al. Quantification of Demodex folliculorum by PCR in rosacea and its relationship to skin innate immune activation. Exp Dermatol. 2012;21:906-10.

21. Hua TC, Chung PI, Chen YJ, Wu LC, Chen YD, Hwang CY, et al. Cardiovascular comorbidities in patients with rosacea: A nationwide case-control study from Taiwan. J Am Acad Dermatol. 2015;73:249-54.

22. Rainer BM, Fischer AH, Luz Felipe da Silva D, Kang S, Chien AL. Rosacea is associated with chronic systemic diseases in a skin severitydependent manner: Results of a case-control study. J Am Acad Dermatol. 2015;73:604-8.

23. Egeberg A, Fowler JF Jr, Gislason GH, Thyssen JP. Nationwide Assessment of Cause-Specific Mortality in Patients with Rosacea: A Cohort Study in Denmark. Am J Clin Dermatol. 2016;17:673-9.

24. Kunutsor SK, Bakker SJ, James RW, Dullaart RP. Serum paraoxonase-1 activity and risk of incident cardiovascular disease: The PREVEND study and metaanalysis of prospective population studies. Atherosclerosis. 2016;245:143-54.

25. Takci Z, Bilgili SG, Karadag AS, Kucukoglu ME, Selek S, Aslan M. Decreased serum paraoxonase and arylesterase activities in patients with rosacea. J Eur Acad Dermatol Venereol. 2015;29:367-70.

26. Edfeldt K, Agerberth B, Rottenberg ME, Gudmundsson GH, Wang XB, Mandal K et al. Involvement of the antimicrobial peptide LL-37 in human atherosclerosis. Arterioscler Thromb Vasc Biol. 2006;26:1551-7

27. Benachour H, Zaiou M, Samara A, Herbeth B, Pfister M, Lambert D, et al. Association of human cathelicidin (hCAP-18/LL-37) gene expression with cardiovascular disease risk factors. Nutr Metab Cardiovasc Dis. 2009;19:720-8.

How to cite this article: Akin Belli A, Altun I, Altun I. Thickness of carotid intima and epicardial fat in rosacea: a cross-sectional study. An Bras Dermatol. 2018;92(6): 820-5. 